\title{
LOSS MODELLING AND EXPERIMENTAL VERIFICATION OF A 98.8\% EFFICIENCY BIDIRECTIONAL ISOLATED DC-DC CONVERTER
}

\author{
Rakesh Ramachandran, Morten Nymand \\ University of Southern Denmark, Odense,Denmark,Email:rar@mmmi.sdu.dk
}

\begin{abstract}
High efficiency in power converters means less power wasted, which implies reduction in heat sink requirement, thereby small in size and less in weight. This power loss reductions in space application leads to smaller solar panels and less fuel on-board, hence reduces the spacecraft size, weight and launch cost. One of the major challenges for power converters in space application is to boost their efficiency, to reduce thermal dissipation problems.
\end{abstract}

In this paper, design and implementation of an ultra-high efficiency isolated bi-directional $\mathrm{dc}-\mathrm{dc}$ converter utilizing GaN devices is presented. Loss modelling of the $\mathrm{GaN}$ converter is also included in this paper. The converter has achieved a maximum measured efficiency of $98.8 \%$ in both directions of power flow, using the same power components. Hardware prototype of the converter along with the measured efficiency curve is also presented in this paper.

\section{INTRODUCTION}

Solar energy is a major source of energy for the satellite in orbit. A bidirectional dc-dc converter is highly desirable to charge the battery with solar energy and in eclipse period to power the satellite equipment.

Some of the mandatory requirement for a converter used in satellite includes high conversion efficiency to reduce thermal dissipation problems, electrical isolation between input and output stages and low mass [1], [2]. Since, the size, reliability and efficiency of the overall system mainly depend on the power losses of the converter; efficiency is a key parameter in designing such a dc-dc converter.

GaN FETs have lower switching loss and conduction loss compared to Si MOSFET. Hence the efficiency of the whole converter can be increased to reduce the thermal dissipation and size, thereby making it more compact [3], [4].

Various bidirectional converters to achieve high efficiency have been discussed in the literature [5]-[7]. In [5], a $2 \mathrm{~kW}, 20 \mathrm{kHz}$ with current doubler topology is presented. The maximum efficiency achieved is $96 \%$ at $600 \mathrm{~W}$ in boost mode and $96 \%$ at $1200 \mathrm{~W}$ in buck mode. A $1 \mathrm{~kW}$ resonant bidirectional converter is presented in
[6] with an efficiency of $97.5 \%$ in forward and $97 \%$ in backward direction.

This paper presents an ultra-high efficiency isolated dcdc converter. GaN devices are used as the switching device to realize the hardware prototype of the converter. This paper also discusses the design of high efficiency magnetics along with design considerations of using $\mathrm{GaN}$ FETs as the switching device. The loss modelling of the $\mathrm{GaN}$ converter is also presented in the paper.

The proposed converter is a bidirectional converter which allows the power flow in both directions using the same power components. The hardware prototype of a $1.7 \mathrm{~kW} \mathrm{GaN} \mathrm{converter} \mathrm{is} \mathrm{presented} \mathrm{in} \mathrm{this} \mathrm{paper.} \mathrm{The}$ measured maximum efficiency of the converter is $98.8 \%$ in both the directions of power flow. The converter has also achieved an efficiency of above $98.5 \%$ over a wide range of output power.

\section{ISOLATED BI-DIRECTIONAL DC-DC CONVERTER}

The circuit diagram of an isolated full bridge bidirectional dc-dc converter is shown in Figure 1.

The converter works as an isolated buck converter in forward direction and isolated boost converter in backward direction. Figure 2 show the operational waveforms of the bidirectional converter in both directions of power flow.

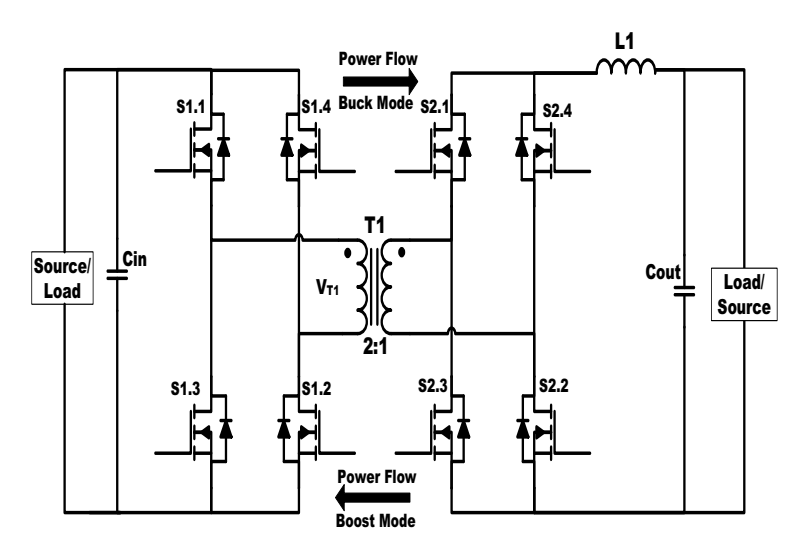

Figure 1. Isolated full-bridge bidirectional dc-dc converter 


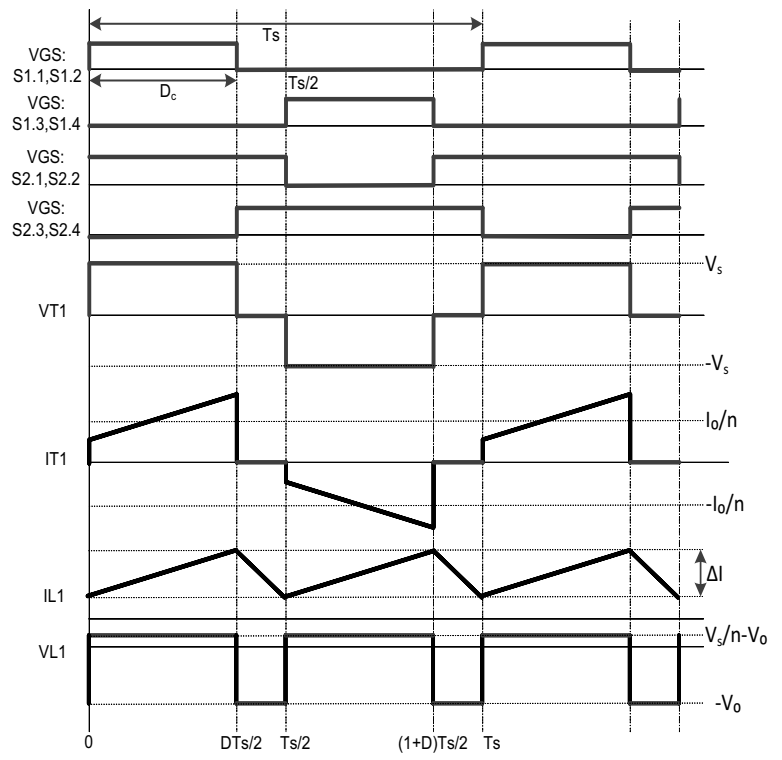

(a).

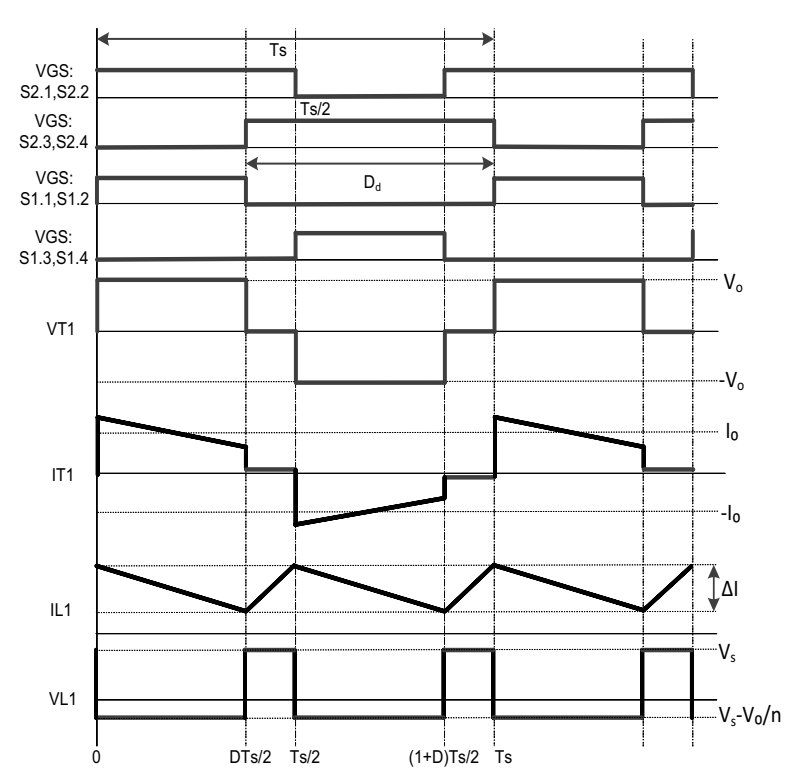

(b).

Figure 2. Operational waveforms of an isolated bidirectional dc-dc converter, (a) buck mode and (b) boost mode

The operation principle of the converter in buck mode and boost mode are explained in [8] and [9], respectively.

\section{CONVERTER DESIGN}

In buck mode of operation, the designed isolated dc-dc converter has an input voltage of $130 \mathrm{~V}$ and output voltage of $52 \mathrm{~V}$. The $130 \mathrm{~V}$ side is referred as the high voltage side and corresponding secondary is referred as the low voltage side. The design specifications of the converter are shown in Table.1.

\begin{tabular}{|l|c|}
\hline Output Power & $1.7 \mathrm{~kW}$ \\
\hline High Voltage Side & $130 \mathrm{~V}$ \\
\hline Low Voltage Side & $52 \mathrm{~V}$ \\
\hline Switching Frequency & $50 \mathrm{kHz}$ \\
\hline Transformer Core & Ferroxcube EE55/28 \\
\hline Inductor Core & Kool M $\mu$ E40 \\
\hline HV side GaN FETs & EPC2010C \\
\hline LV side GaN FETs & EPC2001C \\
\hline
\end{tabular}

Table 1. Bidirectional converter design considerations

\subsection{Transformer}

High frequency transformer galvanic-ally isolates the source and the load. In order to have high efficiency in transformer, both core loss and copper loss has to be reduced. Ferrites E-core has been selected as the core material to have lower core loss. The major challenge to achieve high efficiency in power transformer is to have very low ac-resistance. Extensive interleaving of primary and secondary reduces the proximity effect and hence dramatically reduces the ac-resistance of the transformer
[7]. Extensive interleaving with fewer turns also reduces the leakage inductance of the transformer. Hence, low acresistance and low leakage inductance increases the efficiency of the transformer even at higher power levels.

\subsection{Inductor}

In an isolated dc-dc converter, storage inductor is designed to operate with a dc bias current with a small ac ripple current. Core materials with high saturation flux will help in minimizing the inductor size and increasing the efficiency. Thus, Magnetics Kool M $\mu$ is selected for the inductor core. For high dc bias current application, copper cross section has to be higher; hence copper foils are used for the winding. In order to reduce the acwinding loss, two winding technique, is used for inductor design, explained in [11]. A thin ac winding is used to reduce the ac resistance and a thick dc winding to reduce the dc resistance. Thereby reducing the losses and hence high efficiency in inductor can be realized.

\subsection{GaN FETs}

GaN FETs are used as the switching devices. $200 \mathrm{~V}$ and $100 \mathrm{~V}$ FETs are used at the high voltage and low voltage sides of the transformer respectively. In order to reduce the conduction losses, four devices are used in parallel.

One of the main reasons for selecting GaN device is that they have zero reverse recovery losses compared to $\mathrm{Si}$ MOSFET. This is highly advantageous in low voltage high current synchronous rectification applications. Synchronous rectification helps in improving the efficiency of the overall converter.

The major challenges in using GaN FETs in high power 
converters are the optimum PCB layout design and the design of gate drive circuit. They have a very small threshold voltage and low gate-source voltage rating. A small noise injected into the gate of these devices, due to any parasitic inductance, can cause the device to turn-on or turn-off inadvertently and can cause damage to the whole converter.

LM5114 from Texas Instruments is chosen as the driver for the GaN devices. These drivers are capable of driving multiple GaN FETs due to their large sink current capability. Each driver drives four parallel devices. They also have a split gate output, which provides flexibility to adjust the turn-on and turn-off independently. The driver is compactable with TTL/CMOS signals and can withstand voltages up to $14 \mathrm{~V}$. This allows the driver to provide input directly from any PWM controller. LM5114 has a fast switching speed and minimized propagation delay of $12 \mathrm{nS}$. An LCR tank circuit is usually formed with the parasitic source impedance, gate capacitance and the drive pull down path. This LCR tank circuit causes ringing in the gate of the GaN devices, an optimized resistor is used to damp this ringing [10].

\subsection{Filter capacitors}

Ceramic capacitors are used as the filter capacitors at both high voltage and low voltage sides of the converter. Compared to electrolitic capacitors, ceramic capacitors occupies very small volume. This helps in improving the power density of the converter. Even though the capacitance value in ceramic capacitor reduces with the dc-bias voltage, twice the number of capacitors has been used, which provides enough capacitance at both high voltage and low voltage side of the converter. These capacitors also have a very low ESR value, hence contribute to very low losses.

\section{ANALYTICAL LOSS MODELLING}

Loss modelling of individual power components is performed to predict the performance characteristics of the converter. The losses in the converter are dependent on many functions, mainly on switching frequency, input voltage and load current. The major source of power losses in an isolated converter are distributed among magnetics and the power semiconductor devices.

The power loss in individual components, transformer, inductor and semiconductor devices, can be expressed by a second order quadratic equation as a function of output current, $I_{o}$.

$$
P_{l o s s}=k_{o}+k_{2} I_{o}^{2}
$$

where, $k_{o}$ is the idle losses and $k_{2}$ is the loss factor associated with the resistive losses in the respective power component.
The loss modelling of the individual power components are discussed below.

\subsection{Transformer}

The idle loss in the transformer is mainly the core loss. The core loss can be calculated using the Steinmetz formula [12],

$$
P_{v}=k f^{\alpha} \hat{B}^{\beta}
$$

where, $P_{v}$ is the power loss per unit volume, $B$ is the flux density, $f$ is the frequency, and $k, \alpha, \beta$ are the material parameters.

The ac-resistance of the transformer can be expressed by the equation [9]

$$
R_{a c}=R_{d c} \varphi \frac{\sinh 2 \varphi+\sin 2 \varphi}{\cosh 2 \varphi-\cos 2 \varphi}+\frac{2\left(m^{2}-1\right)}{3} \varphi \frac{\sinh \varphi-\sin \varphi}{\cosh \varphi+\cos \varphi}
$$

where, $\varphi=\mathrm{h} / \delta$, $h$ is the height of the conductor, $\delta$ is the penetration depth, $R_{a c}$ is the ac resistance of the winding, $R_{d c}$ is the winding dc resistance and $m$ is the number of layers in the winding.

The rms current through the primary winding can be written as

$$
I_{R M S, t f r}=\frac{I_{o}}{n} \sqrt{D_{c}}
$$

where, $n$ is the turns ratio and $D_{c}$ is the duty cycle.

The resistive losses in the transformer can be calculated from the ac-resistance and the RMS current through the winding.

The transformer power loss equation can be expressed as a function of output current, $I_{o}$ as

$$
P_{t f r}=2.18+0.00262 I_{o}^{2}
$$

\subsection{Inductor}

Similar to transformer, inductor losses also includes both core loss and winding loss. Core loss can be estimated from the Steinmetz equation as given in (2).

Since the inductor is designed using a two-winding technique, the winding losses includes both ac and dcwinding losses. The losses are calculated assuming $2 / 3$ of the ac ripple current flows through the ac winding and the remaining $1 / 3$ flows through the dc-winding [11]. 
The power loss equation for the inductor is given as

$$
P_{I d r}=0.83+0.001175 I_{o}^{2}
$$

\subsection{GaN FETs}

The losses in GaN FETs include both switching loss and resistive loss.

Gate drive losses and output capacitive losses are included in the switching losses and can be estimated from the equation given below.

Gate drive loss is given by,

$$
P_{\text {drive }}=V_{d} Q_{G} f
$$

where, $V_{d}$ is the drive voltage and $Q_{G}$ is the total gate charge and $f$ is the switching frequency.

The analysis of capacitive switching loss in an isolated $\mathrm{dc}-\mathrm{dc}$ converter is explained in [13]. From [13], the capacitive switching loss in the GaN FETs can be written as,

$$
\begin{gathered}
P_{\text {cap }(\text { loss })}=\left\{2 Q_{o s s, p\left(V_{\text {in }}\right)}-\left[\frac{5}{8} Q_{\text {oss }, p\left(\frac{3 V_{\text {in }}}{8}\right)}+\right.\right. \\
\left.\frac{3}{8} Q_{o s s, p\left(\frac{5 V_{i n}}{8}\right)}\right]+ \\
\left.\frac{2}{n} Q_{o s s, s\left(\frac{V_{i n}}{n}\right)}\right\} V_{\text {in }} f
\end{gathered}
$$

where, $Q_{o s s, p\left(V_{i n}\right)}$ is the output charge of four parallel primary switches at $V_{i n}, Q_{o s s, p\left(\frac{5 V_{i n}}{8}\right)}$ is the output charge of four parallel primary switches at $\frac{5 V_{i n}}{8}, Q_{o s s, p\left(\frac{3 V_{i n}}{8}\right)}$ is the output charge of four parallel primary switches at $\frac{3 V_{\text {in }}}{8}, Q_{o s s, s\left(\frac{V_{i n}}{n}\right)}$ is the output charge at $\frac{V_{\text {in }}}{n}$ of four parallel secondary switches.

Resistive losses in GaN FETs can be estimated from the on-resistance of the respective FETs and the RMS current through them.

Losses in the high voltage FET can be calculated by,

$$
P_{H L O S S}=R_{D S(O N) H} \frac{P_{o} \sqrt{D_{C}}}{n_{c} V_{o}}
$$

where, $R_{D S(O N) H}$ is the on-resistance of high voltage FETs, $P_{o}$ is the output power, and $V_{o}$ is the output voltage in buck mode of operation.

Similarly, the conduction losses in low voltage FET can be expressed as,

$$
P_{L L O S S}=R_{D S(O N) L} \frac{P_{o}}{V_{o}}
$$

where, $R_{D S(O N) L}$ is the on-resistance of low voltage FETs. The power loss equation for the GaN FETs can be written as

$$
P_{\text {GaN }}=3.79+0.00652 I_{o}^{2}
$$

The diectric loss in the filter capacitors can be calculated by,

$$
P_{\text {di_cap }}=2 \pi f C V^{2} \tan \delta
$$

where, $C$ is the filter capacitance value, $V$ is the voltage across the capacitor and $\tan \delta$ is the loss tangent or dissipation factor.

So the total loss in the converter can be expressed as a second order quadratic equation as

$$
P_{\text {tot }}=6.8+0.0117 I_{o}^{2}
$$

From the above loss modelling, efficiency of individual power components are calculated and plotted in Figure 3. Figure also shows the calculated efficiency curve for the converter.

\section{EXPERIMENTAL RESULTS}

The hardware prototype of a $1.7 \mathrm{~kW}$ Isolated bidirectional dc-dc converter is shown in Figure 4. The proposed converter is realized on a PCB having size of $14.3 \mathrm{~cm}$ in length and $7.7 \mathrm{~cm}$ in width.

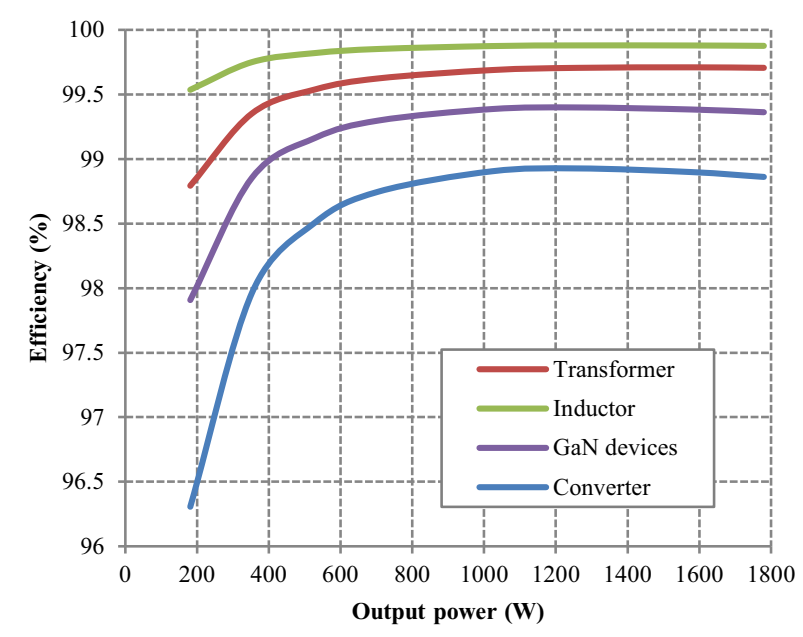

Figure 3.Calculated efficiency curve of the converter along with the efficiency curve of power components 


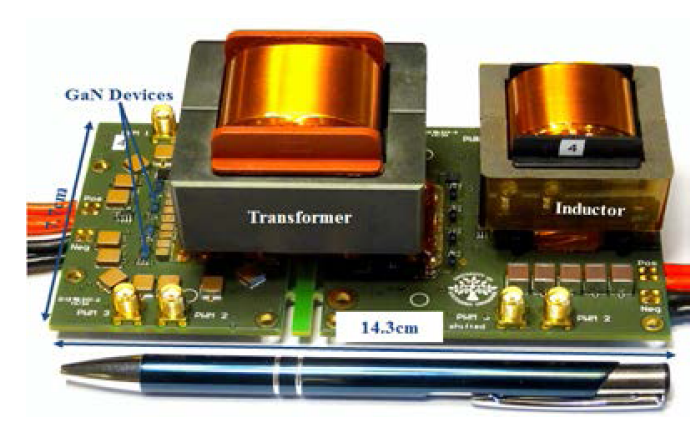

Figure 4. Hardware prototype of the bidirectional isolated $d c-d c$ converter

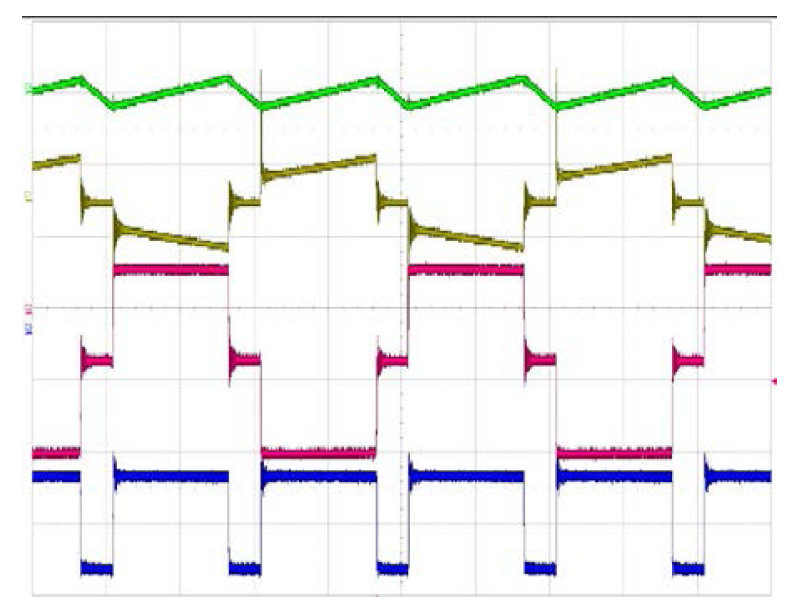

Figure 5. Measured waveforms of the bidirectional $d c-d c$ converter in buck mode of operation (Green: Inductor current 20A/div, Yellow: Transformer primary current 20A/div, Red: Transformer primary voltage 100V/div, Blue: Rectifier voltage 50V/div, Time scale: $5 \mu \mathrm{S} /$ div)

When the efficiency becomes higher, for e.g. above 95\%, extensive care has to be taken to measure the efficiency precisely and accurately. Current measurement is performed using a highly stable and precise sense

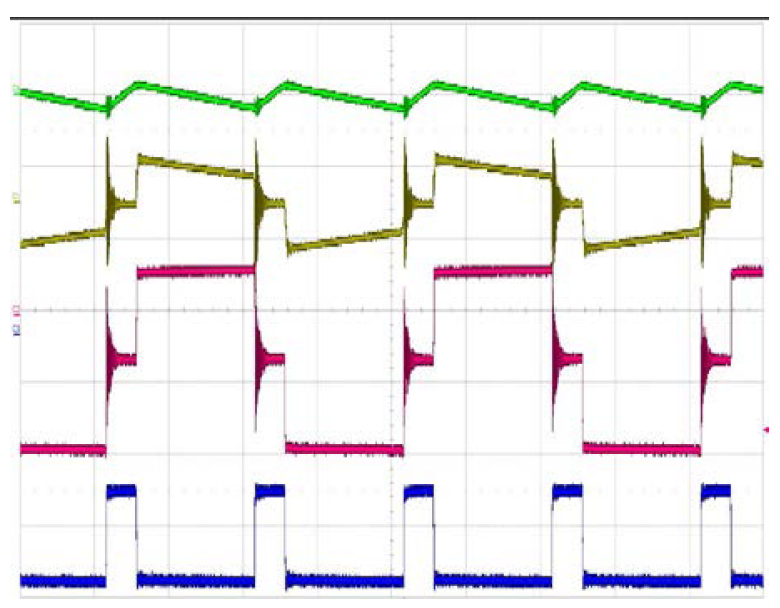

Figure 6. Measured waveforms of the bidirectional dcdc converter in boost mode of operation (Green: Inductor current 20A/div, Yellow: Transformer secondary current 20A/div, Red: Transformer secondary voltage 100V/div, Blue: Rectifier voltage 50V/div, Time scale: $5 \mu \mathrm{S} / \mathrm{div}$ )

resistors and voltage is measured using Keysight's high precision digital multi-meters.

The bidirectional operation of the converter is verified by operating the converter in buck mode and then in boost mode. In buck mode of operation, high voltage side is connected with a voltage source of $130 \mathrm{~V}$ and an electronic load is connected at the low voltage side.

In boost mode of operation, the low voltage side is connected with a voltage source of $52 \mathrm{~V}$ and the high voltage side is connected with an electronic load. The operational waveforms of the converter in both buck and boost mode are measured and shown in Figure 5 and Figure 6, respectively.

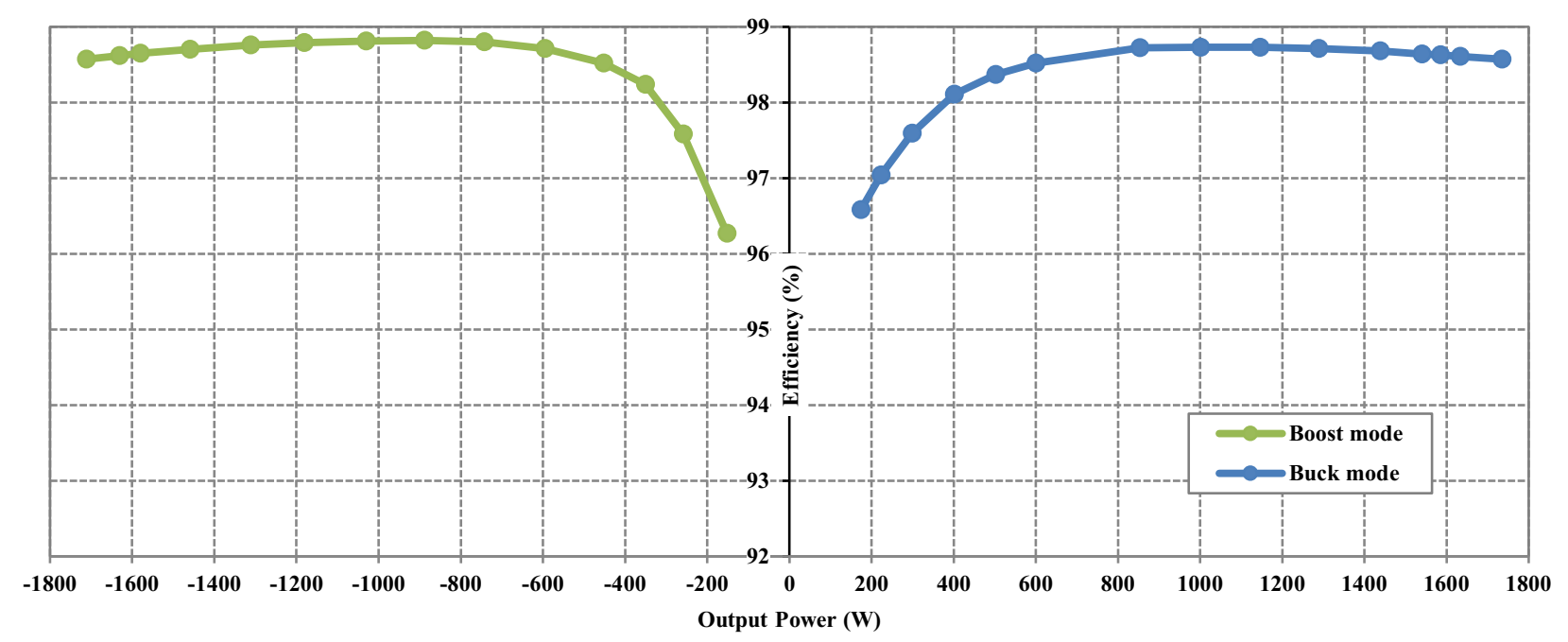

Figure 7. Measured efficiency curve of the bidirectional isolated dc-dc converter 
The efficiency of the converter, both in buck mode and boost mode of operation are also measured and plotted in Figure 7. The isolated bidirectional dc-dc converter has achieved a measured maximum efficiency of $98.8 \%$ in buck mode as well as in boost mode of operation. The converter also exhibited a measured efficiency of above $98.5 \%$ over a wide operating power range, in both directions of power flow.

\section{CONCLUSION}

This paper presents the design and implementation of an isolated bidirectional dc-dc converter. Due to zero reverse recovery losses in GaN FETs, they are used as switching devices to improve the efficiency of the bidirectional dc-dc converter with synchronous rectification.

The design consideration for high efficiency magnetics as well as GaN devices are discussed in this paper. The analytical loss modelling of the individual power components along with calculated efficiency curve for the converter is presented in the paper. The measured efficiency curve of the converter is also shown in the paper. The converter has achieved a measured maximum efficiency of $98.8 \%$ in both buck and boost mode of operation. The efficiency of the isolated bidirectional converter is above $98.5 \%$ over a wide range of output power.

\section{ACKNOWLEDGMENT}

The project is sponsored by the Danish National Advanced Technology Foundation under Intelligent Efficient Power Electronics (IEPE), strategic research center between the industries and universities in Denmark.

\section{REFERENCES}

1. Kankam, M. D. \& Elbuluk, M. E. (2001). A Survey of Power Electronics Applications in Aerospace Technologies. In Proc. 36th Intersociety Energy Conversion Engineering Conference, Savannah, Georgia.

2. Weinberg, A. H. \& Schreuders, J. (1986). A Highpower High-voltage dc-dc Converter for Space Applications. In Proc. IEEE Transactions on Power Electronics, pp.148-160.

3. Kaminski N. (2009). State of the art and the future of wide band-gap devices. In Proc. 13th European Conference on Power Electronics and Applications, pp. 1-9.

4. Millan J. et al. (2014) A survey of wide bandgap power semiconductor devices. In Proc. IEEE Trans. on Power Electronics, pp. 2155- 2163.

5. Chiu, H. J. \& Lin, L. W. (2006). A Bidirectional dc- dc Converter for Fuel Cell Electric Vehicle Driving System. In Proc. IEEE Trans. Power Electron., pp. 950-958.

6. Jiang, T. et al. (2015). A Bidirectional LLC Resonant Converter with Automatic Forward and Backward Mode Transition. In Proc. IEEE Transactions on Power Electronics, pp. 757-770

7. Yu, X. \& Yeaman, P. (2014). A New High Efficiency Isolated Bi-directional dc-dc Converter for DC-bus and Battery-bank Interface. In Proc. 29th Applied Power Electronics Conference and Exposition (APEC), pp. 879-883.

8. Ramachandran, R. et al. (2014). Design of a Compact, ultra-high Efficient Isolated dc-dc Converter utilizing GaN Devices. In Proc. 40th Annual Conference of the IEEE Industrial Electronics Society, IECON, pp. 4256-4261.

9. Nymand, M. \& Andersen, M. A. E. (2010). Highefficiency Isolated Boost dc-dc Converter for Highpower Low-voltage Fuel Cell Applications. In Proc. IEEE Trans. Ind. Electron., pp. 505-514.

10. LM5114 datasheet, Peak current low-side gate driver, (www.ti.com)

11. Nymand, M. et al. (2009). Reducing ac-winding losses in High-current High-power Inductors. In Proc. 35th IEEE IECON Conf., Portugal, pp. 774778.

12. Steinmetz, C. P. (1984). On the Law of Hysteresis. In Proc. IEEE, pp. 197 -221.

13. Ramachandran R. \& Nymand M. (2015). Analysis of Capacitive Losses in GaN Devices for an Isolated Full Bridge DC-DC Converter In Proc. International Conf. on Power Electronics and Drive Systems, pp. 467-472. 